\title{
Spectral properties of magnetohydrodynamic convection with mean horizontal temperature gradient
}

\author{
D. Skandera ${ }^{1}$ and W.-Ch. Müller ${ }^{1}$ \\ ${ }^{1}$ Max-Planck-Institut für Plasmaphysik, 85748 Garching, Germany \\ email: dss@ipp.mpg.de, Wolf.Mueller@ipp.mpg.de
}

\begin{abstract}
Spectral properties of convective magnetohydrodynamic (MHD) turbulence in two and three dimensions are studied by means of direct numerical simulations (Skandera D. \& Müller W.-C. 2006). The investigated system is set up with a mean horizontal temperature gradient in order to avoid a development of elevator instabilities in a fully periodic box. All simulations are performed without mean magnetic field. The applied resolution is $512^{3}$ and $2048^{2}$. The MHD equation are solved by a numerical code (Müller \& Biskamp 2000) that uses a standard pseudospectral scheme. For removing of aliasing errors a spherical truncation method is employed. Obtained results are compared with predictions of various existing phenomenological theories for magnetohydrodynamic and convective turbulence (Müller \& Biskamp 2000). While the three-dimensional system is found to operate in a Kolmogorov-like regime where buoyant forces have a negligible impact on the turbulence dynamics (relatively low Rayleigh number achieved in the simulation; $\mathrm{Ra} \sim 10^{6}$ ), the two-dimensional system exhibits interesting irregular quasi-oscillations between a buoyancy dominated Bolgiano-Obukhov-like regime of turbulence and a standard Iroshnikov-Kraichnan-like regime of turbulence (Müller \& Biskamp 2000). The most important parameter determining the turbulent regime of $2 \mathrm{D}$ magnetoconvection, apart from a high Rayleigh number, seems to be the mutual alignment of velocity and magnetic fields. The non-linear dynamics and the interplay between individual fields are examined with different transfer functions that confirm basic assumptions about directions of energy transfer in spectral space. Kinetic, magnetic and temperature energy are transported by a turbulent cascade from large to smaller scales. The local/nonlocal character of the transport is tested for several individual terms in the governing equations. Moreover, other statistical quantities, e.g. probability density functions, are computed as well. A passive character of the temperature field in the investigated three-dimensional magnetoconvection is supported by computations of intermittency using extended self-similarity. The intermittency of the Elsasser field $z^{+}$is in agreement with results from numerical simulations of isotropic MHD turbulence (Müller \& Biskamp 2000). The intermittency of the temperature field is found to approximately agree with results of passive scalar measurements in hydrodynamic turbulence (Ruiz-Chavarria, Baudet \& Ciliberto 1996).
\end{abstract}

Keywords. Convection, magnetohydrodynamics: MHD

\section{References}

Müller, W.-C. \& Biskamp, D. 2000, Phys. Rev. Lett. 84, 475

Ruiz-Chavarria, G., Baudet, C., \& Ciliberto, S. 1996, Phys. D 99, 369

Skandera D. \& Müller W.-C. 2006, in preparation 